\title{
The Trifold Universe
}

\author{
Henry Gu Cao, Zhiliang Cao \\ Northwestern University, Evanston, IL, USA \\ Email: henrycao2018@u.northwestern.edu
}

How to cite this paper: Cao, H.G. and Cao, Z.L. (2018) The Trifold Universe. Open Access Library Journal, 5: e4292. https://doi.org/10.4236/oalib.1104292

Received: December 27, 2017

Accepted: March 4, 2018

Published: March 7, 2018

Copyright $\odot 2018$ by authors and Open Access Library Inc.

This work is licensed under the Creative Commons Attribution International License (CC BY 4.0).

http://creativecommons.org/licenses/by/4.0/

\begin{abstract}
This paper studies a new concept called universal folding. The visible universe is the result of a complex invisible folding series. There are three major folds (Trifold): from the Plank length, to the radius of an electron, and finally, to the radius of universe. Each of the three-major folds follows the same folding formula. Our findings help us understand the grand architecture of the universe. The ideal average human height, city block size, city size, diameter of the continents, the diameter of the Sun, the distance between the Earth and the Sun, the radius of the solar system, the radius of the Milky Way galaxy, the average diameter of the filament of the universe, and the radius of the universe are important resonance lengths in the formula. This paper helps scientists study everything from a new angle and become aware of the architectural design of the universe.
\end{abstract}

\section{Subject Areas}

Computational Physics, Theoretical Physics

\section{Keywords}

Cosmic Ray, Black Hole, Astronomy, Dark Matter, Gravity, Unified Field Theory

\section{Introduction}

The Plank length, diameter of the electron, and size of the universe are three important constants. The three constants are inter-related. The numerical relationships of these three constants are very interesting. They are the experimental base for the paper.

The Plank Length is the smallest length among three constants. To simplify the study, instead of Plank Length, Torque Grid size is used as the unit of measurement. There are two constants as follow:

The size of electron: 


$$
r_{e}=1.0390872 \times \pi^{2} \times 137^{9} / 2^{1 / 2}
$$

The size of the universe:

$$
r_{u}=\left(137 \times r_{e}^{3}\right) /(1.0397 \times \pi)
$$

Based on the Equations (1)-(2), the number 137 is an important factor. The number 137 happens to be the number of twisting steps of the election [1] [2]. The above formula indicates that the Torque Grid itself is the result of 137-step twist movements as well. Furthermore, the paper establishes following model:

The world is built on the freedom of three dimensional movements [2]. The freedom of the movements creates the fundamental building block. 137 steps [1] [2] complete a twisting cycle in three-dimensional space. This is the basic unit of space-time as a result of the movements. In Unified Field Theory [1]-[13], the building block is called the Torque Grid.

The Torque Grid twisting movement continues because a twisting movement is an open system. The end of the movements is the starting point of the next block. The movements continue. When the twisting movements complete a cycle, the inward/outward movement is one step. 137-step twisting cycle creates a new unit:

$$
137 \times 137 \times 137 \times \cdots
$$

Basic three-dimensional concepts include the line, circle, cube and sphere. The folding has to deal with the line and cycle on top of 137 twisting steps. Topologically, the circular features are reflected factors of $\pi$ and $2^{1 / 2}$. The folding continues to form the electron as Equation (1).

Imaging that an electron is a line, $r_{e}^{3}$ forms a cube:

$$
r_{e}^{3}
$$

The electron cube twists 173 times more to have strong forces; the straight length is the size of universe as Equation (2).

Now, the above model provides explanation of the numerical relationships of the Plank length, diameter of the electron, and size of the universe.

The paper will draw additional details of the universe as it continues the interesting predictions.

\section{Results}

\subsection{Folding of Electron}

The Torque Grids line up in a 137 -unit straight line, then $137 \times 137$ square, next $137 \times 137 \times 137$ cube (Figure 1 ).

The above folding model (Figure 1 ) is the $1^{\text {st }}$ level folding. The folding (Table 1) repeats as follow:

\subsection{Electron Size Black Hole Core and the Universe}

In the previous chapter, the electron energy can be folded into a cube with height of $137^{3}$ Torque Grid size. The cube occupied and distorted space (Table 2). 


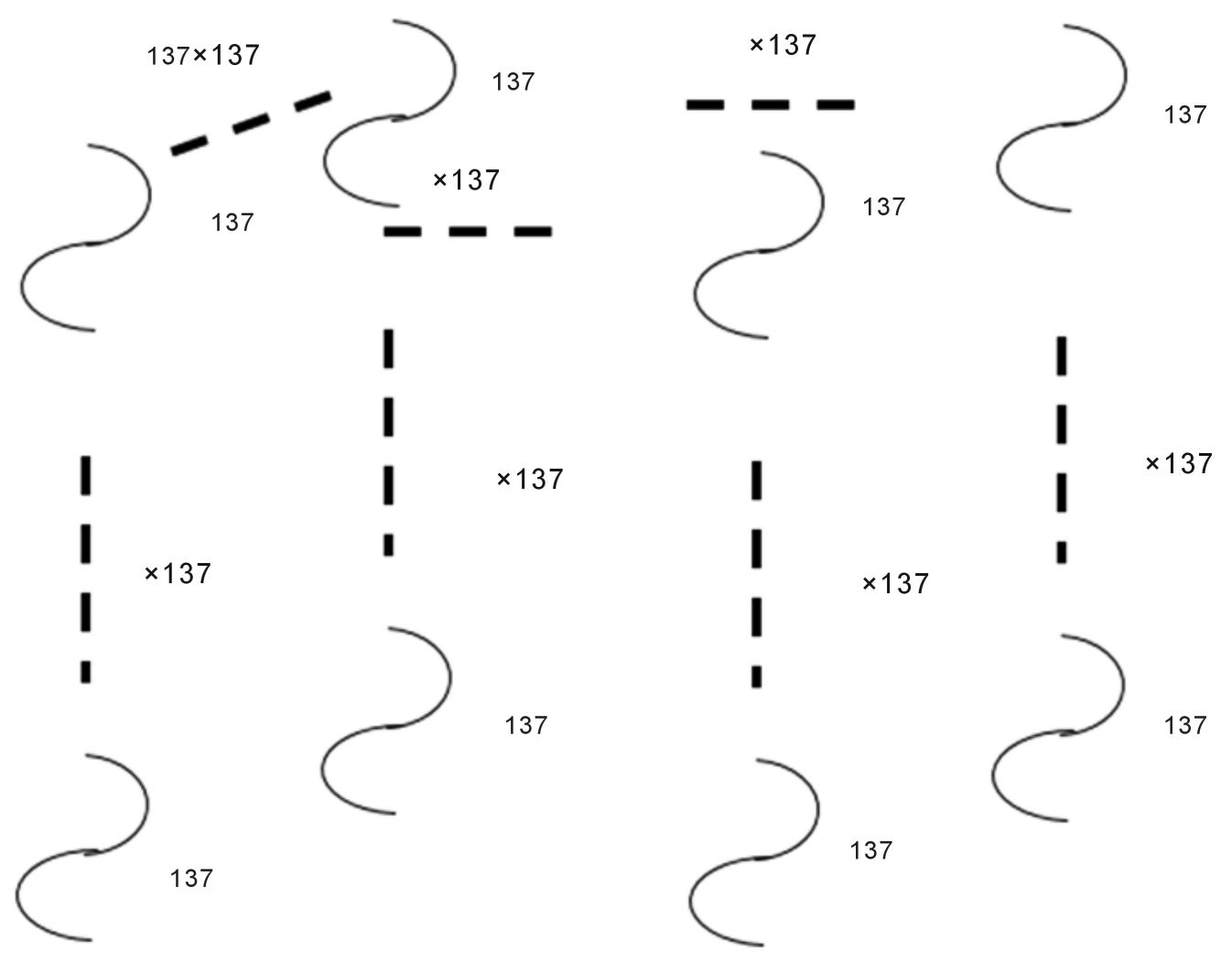

Figure 1. Folding model.

Table 1. Electron folding.

\begin{tabular}{cccc}
\hline Step Count & Folding & \\
\cline { 2 - 4 }$\pi / 2^{1 / 2} \times 137^{3}$ & Folding Cube & Cube Height & Meaning \\
\hline$\pi / 2^{1 / 2} \times 137^{6}$ & $137 \times 137 \times 137$ & 137 & $1^{\text {st } l e v e l ~ c u b e ~}$ \\
$1.0390872 \times \pi^{2} \times 137^{9} / 2^{1 / 2}$ & $137^{2} \times 137^{2} \times 137^{2}$ & & $137^{2}$ \\
& $137^{3} \times 137^{3} \times 137^{3}$ & $137^{3}$ & Total steps cube are Electron $/ 1.04 \pi$
\end{tabular}

Note: for a sphere, surface twisting steps: $\pi \times 1.0390872$; Torque Grid size: $D=2.2856509 \times 10^{-35} \mathrm{~m}$; The radius of an electron: $r_{e}=1.0390872 \times \pi^{2} \times 137^{9} / 2^{1 / 2} D=2.81794 \times 10^{-15} \mathrm{~m}$.

Table 2. Blackhole and universe folding.

\begin{tabular}{cccc}
\hline Case & Radius & Folded Cube size & Steps \\
\hline Electron & $r_{e}=1.0390872 \times \pi^{2} \times 137^{9} / 2^{1 / 2}$ & $137 \times 137 \times 137$ & $S_{e}=1.0390872 \times \pi^{2} \times 137^{9} / 2^{1 / 2}$ \\
$\begin{array}{c}\text { Black Hole at the radius } \\
\text { of an electron }\end{array}$ & $r_{e}$ & $1.0390872 \times \pi^{2} \times 137^{9} / 2^{1 / 2}$ & $1.03845 \times 1.0390872$ \\
& & & $\times\left(137 \times \pi \times\left(\pi \times 137^{9} / 2^{1 / 2}\right)^{3}\right) \pi$ \\
Universe & $r_{u}=1.03845 \times 1.0390872$ & $1.03845 \times 1.0390872$ & $r_{u} \times s_{e} \times s_{e}($ Proximity $)$ \\
& $\times\left(137 \times \pi \times\left(\pi \times 137^{9} / 2^{1 / 2}\right)^{3}\right) \pi$ & $\times\left(137 \times \pi \times\left(\pi \times 137^{9} / 2^{1 / 2}\right)^{3}\right) \pi$ & \\
\hline
\end{tabular}


Electron folding ends the sub-atomic level [11]-[26] folding. Next folding level uses electron size as its unit. The folding unit changes with the context as it is relative to the current folding structure.

The total steps in the above formula is the size of the universe:

$$
r_{u}=1.03845 \times 1.0390872 \times\left(137 \times \pi \times\left(\pi \times 137^{9} / 2^{1 / 2}\right)^{3}\right) \pi D=60 \mathrm{BLY}
$$

In the later chapter, the Black Hole (BH) core mass has the following formula:

$$
\left(1.03845 \times 137 \times\left(\pi \times 137^{6}\right)^{3 / 2}\right) \times m_{e}
$$

where, $m_{e}$ is mass of an electron.

\subsection{Galaxy Filament}

Based on folding formula Equation (3), the next substructure size:

$$
60 \mathrm{BLY} /(\pi \times 137 \times 1.03845)=134 \mathrm{MLY}
$$

The average galaxy filament void's diameter is 134MLY.

When multiple void spheres with the same diameter form a dense structure, face central crystal (FCC) is one of the understandable configurations.

Two pairs of interacting particles are arranged in the following (Figure 2) [27] [28] manner:

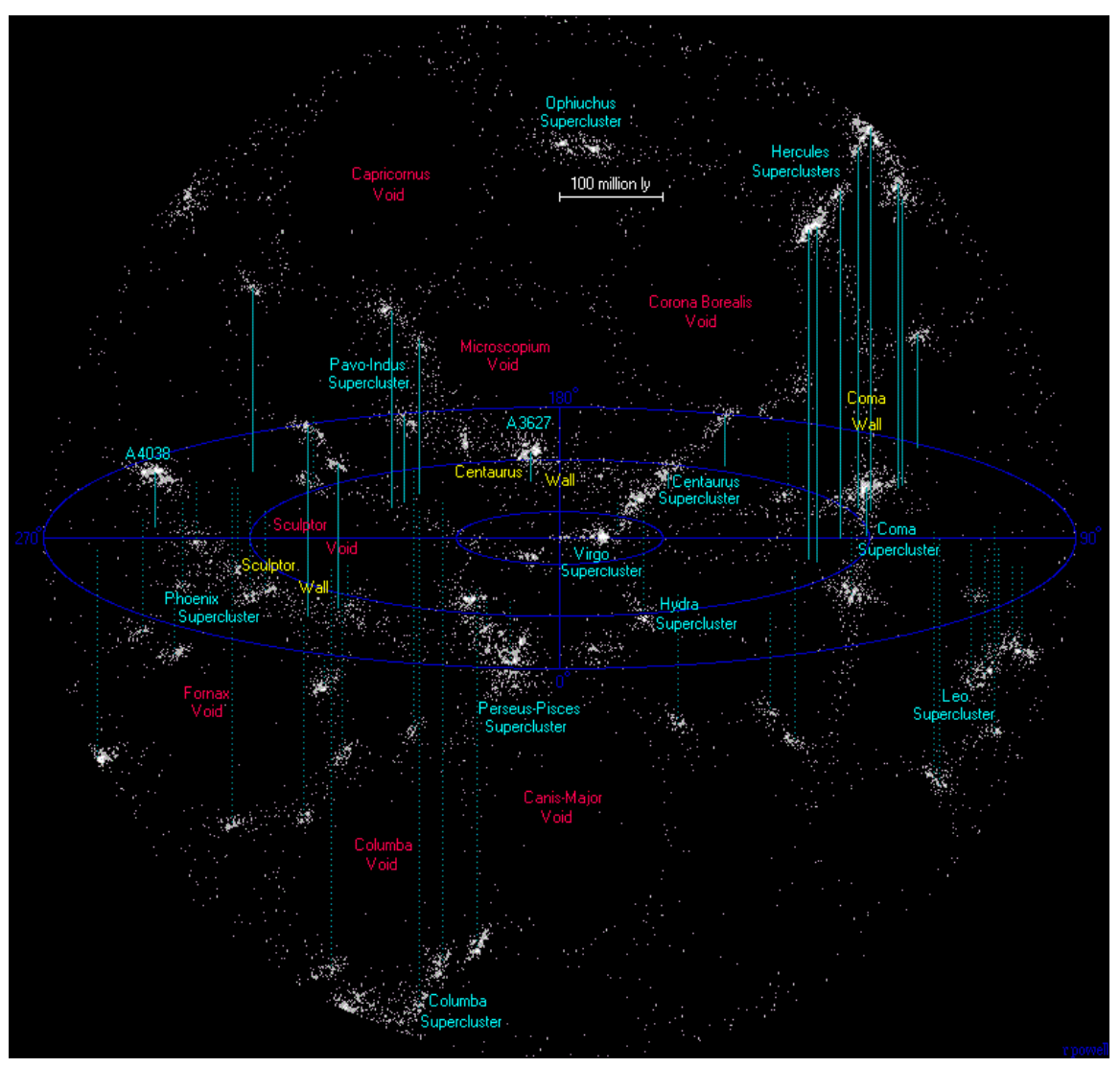

Figure 2. Galaxy filament. 


\subsection{Resonance Length and Human}

Humans have different heights and live in villages/cities in different shapes and sizes. The folding of the universe is a physics principle, not societal. Even though average human height has changed over the millennia, this paper proposes a specific human height as the "ideal" height derived from universal folding. In other words, the "ideal" height is an equilibrium height even though variations exist in reality. Everything varies, such as size of continent, size of the cities, ...

What can the physics figures do? Physics provides reference numbers to guide our practices in urban planning and future predictions.

The resonance length of:

$$
\pi / 2^{1 / 2} \times 137^{9} \times \pi / 2^{1 / 2} \times 137^{9} \times \pi / 2^{1 / 2} \times 137 \times D=9922.1 \mathrm{~km}
$$

The circumference of the earth:

$$
40.075 \mathrm{~km}=4.04 \times 9922.1 \mathrm{~km}
$$

The factor 4 represents equatorial division of the world: America continent, Europe/African, Asia/Australia, and Pacific Ocean.

The land divisions are Asia, Australia, Europe, Africa, North America, and South America. The future world may be united, while six major regions may still exist.

The resonance length of:

$$
\pi / 2^{1 / 2} \times 137^{9} \times \pi / 2^{1 / 2} \times 137^{9} \times \pi / 2^{1 / 2} \times D=72.424 \mathrm{~km}=45 \text { miles }
$$

An ideal city size can be 45 miles.

The resonance length of:

$$
\pi / 2^{1 / 2} \times 137^{9} \times \pi / 2^{1 / 2} \times 137^{8} \times D=237.974 \mathrm{~m}
$$

In square shape, it occupies 14 acres. It is the ideal city block size.

The resonance length of:

$$
\pi / 2^{1 / 2} \times 137^{9} \times \pi / 2^{1 / 2} \times 137^{7} \times D=1.737 \mathrm{~m}=5 \text { feet } 8.3871 \text { in }
$$

Ideal average height of the human.

\subsection{Folding Table}

See Table 3.

Table 3. Folding table.

\begin{tabular}{cccc}
\hline Sequence & Length $(\mathrm{m})$ & Folding & Meaning \\
\hline$\pi / 2^{1 / 2 \star} 137^{3}$ & $1.3 \times 10^{-28}$ & $1^{\text {st }}$ plate & $1^{\text {st }}$ plate \\
$137^{3}$ & $3.36 \times 10^{-22}$ & $2^{\text {nd }}$ plate & $2^{\text {nd }}$ plate \\
$137^{3}$ & $8.63 \times 10^{-16}$ & $3^{\text {rd }}$ plate & Electron $/ .04$ \\
$\pi / 2^{1 / 2 \star} 1.039^{\star} 137^{2}$ & $3.7 \times 10^{-11}$ & $\pi^{2} / 2^{\star} 137^{11}$ & $1 / 2$ Carbon \\
$137^{3}$ & $9.56 \times 10^{-5}$ & $\pi^{2} / 2^{\star} 137^{14}$ & Cell \\
$137^{2}$ & 1.8 & $\pi^{2} / 2^{\star} 137^{16}$ & Human Height \\
137 & 247 & $\pi^{2} / 2^{\star} 137^{17}$ & City Block \\
$137^{\star} \pi / 2^{1 / 2}$ & $75.2 \times 10^{3}$ & $\pi^{2} / 2^{3 / 2 \star} 137^{18}$ & City Size \\
\hline
\end{tabular}


Continued

\begin{tabular}{cccc}
\hline 137 & $10.3 \times 10^{6}$ & $\pi^{3} / 2^{3 / 2 *} 137^{19}$ & Continent \\
137 & $1.41 \times 10^{9}$ & $\pi^{3} / 2^{3 / 2 *} 137^{20}$ & Sun Diameter \\
137 & $1.93 \times 10^{11}$ & $\pi^{3} / 2^{3 / 2 *} 137^{21}$ & Sun to Earth \\
137 & $26.5 \times 10^{12}$ & $\pi^{3} / 2^{3 / 2 *} 137^{22}$ & Solar System \\
$137^{4}$ & $9.3 \times 10^{21}$ & $\pi^{3} / 2^{3 / 2 *} 137^{26}$ & Galaxy \\
137 & $135 \mathrm{MLY}$ & $\pi^{3} / 2^{3 / 2 *} 137^{27}$ & Galaxy Filament \\
$1.04^{*} 137 \pi$ & $60 \mathrm{BLY}$ & $1.04^{*} \pi^{4} / 2^{3 / 2 *} 137^{28}$ & Universe \\
\hline
\end{tabular}

Note: In the above folding table, Torque Grid size is unit.

\subsection{Particle Folding}

Electrons have a gravitational mass wave length resonance, energy charged distortion resonance, and energy folding resonance. Electron energy becomes a particle folding unit, while the size of the electron becomes the particle size.

The electron has a single Torque Grid line. Similar to an electron, a fundamental wave [3] in a particle has a single Torque Grid line as well.

A proton has the following folding formula:

$$
(2 \times 3 \times 5) \times(2 \times 3 \times 5)+(2 \times 3 \times 5) \times(2 \times 3 \times 5)+(2 \times 3 \times 5)+2 \times 3
$$

Since $2 \times 3$ and $2 \times 3 \times 5$ are stable energy interaction formations. They are both fundamental waves [3] and each has single Torque Grid line. The distorted Torque Grids in each line have different formations. The fundamental wave $2 \times 3$ has a plate formation inside the grid, while $2 \times 3 \times 5$ has a cube formation instead.

The Torque Grid within $(2 \times 3 \times 5) \times(2 \times 3 \times 5)$ line has a cube formation as well. $(2 \times 3 \times 5)$ cube builds on top of $(2 \times 3 \times 5)$ cube.

In [3], proton has three axes, $(2 \times 3 \times 5) \times(2 \times 3 \times 5),(2 \times 3 \times 5) \times(2 \times 3 \times 5)$, and $(2 \times 3 \times 5)$.

When a neutron and a proton interact, the third axis becomes $2 \times 2 \times 4$ as the wave factor "2" interacts between two particles (proton and neutron) and the Torque lines in the particle center is wrapped with " 2 " wave as well:

$$
(2 \times 3 \times 5) \times(2 \times 3 \times 5)+2 \times 2 \times 4+2 \times 3
$$

Helium:

$$
4 \times\left(2(2 \times 3 \times 5)^{2}+2 \times 2 \times 4+2 \times 3\right)+2 \times 3
$$

Omitting the axes structural components, $(2 \times 3 \times 5)^{2}$ and $2 \times 2 \times 4$, the main structural components $[3,4]$ are (Table 4).

\section{Calculations}

\subsection{Size of Electron}

Size of electron:

$$
r_{e}=2.81794 \times 10^{-15} \mathrm{~m}
$$


Table 4. Particle folding table.

\begin{tabular}{|c|c|c|c|}
\hline Element & Z & NMR Properties & Mass in Atomic Unit (u) \\
\hline $\mathrm{He}$ & 2 & $(\mathrm{Z}+\mathrm{N})\left(2^{\star} 3\right)+2$ & 1823.574845 \\
\hline $\mathrm{Li}$ & 3 & $(Z+N)\left(2^{\star} 3\right)+2^{\star} 3$ & 1826.979364 \\
\hline $\mathrm{Be}$ & 4 & $(\mathrm{Z}+\mathrm{N})\left(2^{\star} 2+2^{\star} 3\right)+2+3$ & 1824.911461 \\
\hline B & 5 & $(\mathrm{Z}+\mathrm{N})\left(2^{\star} 2+2^{\star} 2\right)+3+4$ & 1823.976003 \\
\hline $\mathrm{C}$ & 6 & $(\mathrm{Z}+\mathrm{N})\left(2^{\star} 3\right)+2^{\star} 2$ & 1822.388485 \\
\hline $\mathrm{N}(15)$ & 7 & $15(2 \star 3)+2 \star 3$ & 1822.788739 \\
\hline $\mathrm{O}$ & 8 & $4\left(2^{\star} 3\right)+12(2+3)+2^{\star} 2$ & 1821.809104 \\
\hline $\mathrm{F}$ & 9 & $(\mathrm{Z}+\mathrm{N})\left(2^{\star} 3\right)+2^{\star} 2$ & 1822.261603 \\
\hline $\mathrm{Ne}$ & 10 & $(\mathrm{Z}+\mathrm{N})(2+3)+2^{\star} 3$ & 1821.699449 \\
\hline $\mathrm{Na}$ & 11 & $17(2+3)+6\left(2^{\star} 3\right)+3+4$ & 1821.599378 \\
\hline $\mathrm{Mg}$ & 12 & $(\mathrm{Z}+\mathrm{N})(2+3)+2 * 3$ & 1821.252346 \\
\hline $\mathrm{Al}$ & 13 & $(\mathrm{Z}+\mathrm{N})(2+3)+2^{\star} 2$ & 1821.160595 \\
\hline $\mathrm{Si}$ & 14 & $20(2+3)+8\left(2^{\star} 2\right)+2^{\star} 2$ & 1820.886329 \\
\hline $\mathrm{P}$ & 15 & $20(2+3)+11\left(2^{\star} 2\right)+2^{\star} 3$ & 1820.861722 \\
\hline$S$ & 16 & $13\left(2^{\star} 2\right)+19(2+3)+2^{\star} 3$ & 1820.828752 \\
\hline $\mathrm{Fe}(56)$ & 26 & $45\left(2^{\star} 2\right)+11(2+3)+2^{\star} 3$ & 1820.306312 \\
\hline $\mathrm{Fe}(57)$ & 26 & $45\left(2^{\star} 2\right)+14(2+3)+2 \star 3$ & 1820.366212 \\
\hline
\end{tabular}

Torque Grid size:

$$
D=2.2856509 \times 10^{-35} \mathrm{~m}
$$

The formula calculates the exact size of the electron,

$$
\left(1.039087 \times \pi^{2} \times 137^{9} / 2^{1 / 2}\right) \times 2.2856509 \times 10^{-35}=2.81794 \times 10^{-15} \mathrm{~m}
$$

\subsection{Composition of the Core in Black Hole}

A Black Hole $(\mathrm{BH})$ follows the following equation:

$$
M=(1 / 2) R C^{2} / G
$$

The mass of the $\mathrm{BH}$ when the radius is same as the proton,

$$
\begin{aligned}
M & =0.5 \times 0.86325 \times 10^{-15} \times\left(2.99752458 \times 10^{8}\right)^{2} / 6.67808 \times 10^{-11} \\
& =5.8074 \times 10^{11} \mathrm{~kg}
\end{aligned}
$$

The mass of electron is:

$$
9.10938356 \times 10^{-31}
$$

In unit of electron mass:

$$
M=6.3752 \times 10^{41}=1.03845 \times 137 \times\left(\pi \times 137^{6}\right)^{3} / 2
$$




\subsection{Physics Constants}

Charged energy causes one Torque Grid twisting distortion at the surface of the electron. Based on the previous chapters, for given Torque Grid size $D$, the radius of electron:

$$
r_{e}=1.0390872 \times \pi^{2} \times 137^{9} / 2^{1 / 2} D
$$

Inside the radius of electron,

$$
\left(1.03845 \times 137 \times\left(\pi \times 137^{6}\right)^{3 / 2}\right)
$$

Times electron mass (me) will create distortion of $r_{e}$. Since,

$$
M=(1 / 2) R C^{2} / G
$$

Therefore,

$$
\begin{aligned}
& G=(1 / 2) R C^{2} / M \\
& =(1 / 2)\left(1.0390872 \times \pi^{2} \times 137^{9} / 2^{1 / 2}\right) D C^{2} /\left(1.03845 \times 137 \times\left(\pi \times 137^{6}\right)^{3 / 2}\right) \times m_{e} \\
& =1.0006136 D C^{2} / \pi \times 137^{10} \times 2^{1 / 2} \times m_{e}
\end{aligned}
$$

The above equation can calculate the Gravitational Constant. Equation,

$$
\begin{gathered}
D=\left(G h / \pi c^{3}\right)^{1 / 2} \\
h=D^{2} \times \pi c^{3} / G
\end{gathered}
$$

Can calculated Planck Constant.

Since energy is defined as twisting distortion of the space, following is relationship between distortion, mass, and force:

$$
F=m c^{2} \times(\mathrm{d} D / \mathrm{d} s)
$$

All the fundamental Physics constants can be calculated with the above equations.

\section{Discussion}

\subsection{Folding Model}

The folding is evident in the electron-sized blackhole where the space is completely filled in with distorted Torque Grids in a hierarchical structure (Figure 1):

Lined up Grid cube forms a line

Rows of lines form a square sheet

Layers of square sheets form a cube

Lined up cube form a line

$\cdots$

When the above folding process, from cube to cube, repeats three times, a balanced cube A $(137 \times 137 \times 137)$ is formed. The unfolded cube A stretches to the size of the electron $\left(137^{3} \times 137^{3} \times 137^{3}\right)$. For an electron, the distorted Torque Grid does not fold. The Torque Grids line up to form the electron. 
When each Grid 137 times, it forms a strong force distortion.

Use cube $A$ as base cube, and repeat cube to cube folding six-times, a balanced cube $B\left(137^{3} \times 137^{3} \times 137^{3}\right)$ is formed. When each grid is strong force distortion, the cube B becomes $137 \times 137^{3} \times 137^{3} \times 137^{3}$. The cube B fills up the space of electron, and the unfolded cube $B$ stretches to the size of the universe $\left(137 \times 137^{9} \times 137^{9} \times 137^{9}\right)$. The mass of cube B blackhole makes itself blackhole.

\subsection{Human and Physics Constants}

Between the size of the electron and size of the visible universe, not everything is folded like blackhole. Even though the paper uses extreme blackhole case to demonstrate the reasoning of the folding model, the folding model is not yet validated via a physical device. The paper finds something that is familiar to everyone to demonstrate the folding model. The theoretical human height is in the folding table. It means that inside of the electron size blackhole, the human height is associated with a stable folding structure. The precision of the folding model predictions will convince the readers. It is impossible to explain everything using a simple folding model. Therefore, try not to do that.

The theoretical human height can be calculated using a physics equation gives everyone an answer and additional questions. It demonstrates that the folding model has direct impact on human society. The proposed model suggests that the invisible hand of the folding model does shape human life and the destiny of humanity. We are free to disagree, but these predictions made by my models will inevitably reveal the equilibrium that humanity is marching towards.

\subsection{Unexplained Constants}

In the formula of the universe folding steps:

$$
1.03845 \times 1.0390872 \times\left(137 \times \pi \times\left(\pi \times 137^{9} / 2^{1 / 2}\right)^{3}\right) \pi
$$

The constant 1.03845 and 1.0390872 are not fully explained. Further research may yield answers to this issue.

Notice that approximately,

$$
1.03845=27 / 26
$$

It is possible that the above formula has some special meaning.

In the formula of Gravitational constant:

$$
G=1.0006136 D C^{2} / \pi \times 137^{10} \times 2^{1 / 2} \times m_{e}
$$

The constant

$$
1.0006136=1+1 / 1629.72
$$

The constant is close to one. On the first look, it is not very attractive. Giving explanation to the above constant will surely bring progress to the theory this paper proposes. 


\section{Conclusions}

The numerical relationships of the three constants, Planck Length, diameter of the electron, and size of the universe, provide important insights.

Based on the calculation results, the paper proposes a universal folding model that explains how the universe is built.

There is no smallest building block in the universe. To simplify the study, the model starts with the building block called Torque Grid as result of random movements. As the movements continue outward/inward, the building blocks are connected by the movements. The connections/pilings are in form of line, circle, cube, and sphere. The paper simply models the formation as "folding" structure.

The numerical ratios of the three constants predict that the Torque Grids are folded into an electron. Next, the electrons are folded into particle and universe. The major folding points are: Human Height, Length of a City Block, the Diameter of a City, Diameter of a Continent, Diameter of the Sun, Distance from the Sun to Earth, Diameter of the Solar System, Diameter of a Galaxy, Diameter of a Galaxy filament, and Diameter of the Universe.

Even though there is no standard human height, the predicted "ideal" height for human is a physics constant.

As more physicists begin to understand the meaning the universal folding, more useful predictions will be made.

\section{Acknowledgements}

Mark L. Trainer thanks for revising the paper and be a loyal reader.

\section{References}

[1] Cao, Z.L. and Cao, H.G. (2013) Unified Field Theory. American Journal of Modern Physics, 2, 292-298. https://doi.org/10.11648/j.ajmp.20130206.14

[2] Cao, Z.L., Cao, H.G. and Qiang, W.A. (2015) Theory of Everything. Frontiers of Astronomy, Astrophysics and Cosmology, 1, 31-36.

[3] Cao, Z.L. and Cao, H.G. (2013) Unified Field Theory and the Configuration of Particles. International Journal of Physics, 1, 151-161.

[4] Cao, Z.L. and Cao, H.G. (2014) Unified Field Theory and Topology of Nuclei. International Journal of Physics, 2, 15-22. https://doi.org/10.12691/ijp-2-1-4

[5] Cao, Z.L. and Cao, H.G. (2016) Gravitational Wave and Transparent Crystal Black Hole. Open Access Library Journal, 3, e3038. https://doi.org/10.4236/oalib.1103038

[6] Cao, Z.L., Cao, H.G. and Qiang, W.A. (2015) Unified Field Theory and Topology of Atom. American Journal of Modern Physics, 4, 1-7.

[7] Cao, Z.L. and Cao, H.G. (2014) Cubic Atom and Crystal Structures. International Journal of Physics, 2, 277-281. https://doi.org/10.12691/ijp-2-6-11

[8] Cao, Z.L. and Cao, H.G. (2014) Unified Field Theory and Foundation of Physics. International Journal of Physics, 2, 158-164. https://doi.org/10.12691/ijp-2-5-5

[9] Cao, Z.L. and Cao, H.G. (2013) Unified Field Theory and the Hierarchical Universe. 
International Journal of Physics, 1, 162-170.

[10] Cao, H.G. and Cao, Z.L. (2013) Drifting Clock and Lunar Cycle. International Journal of Physics, 1, 121-127.

[11] Cao, Z. and Gu Cao, H. (2013) Non-Scattering Photon Electron Interaction. Physics and Materials Chemistry, 1, 9-12.

[12] Cao, Z. and Gu Cao, H. (2013) SR Equations without Constant One-Way Speed of Light. International Journal of Physics, 1, 106-109.

[13] Cao, H., Cao, Z. and Qiang, W. (2015) Nuclear Lattices, Mass and Stability. Open Access Library Journal, 2, 1-22. https://doi.org/10.4236/oalib.1101504

[14] Myers, E.G., Wagner, A., Kracke, H. and Wesson, B.A. (2015) Atomic Masses of Tritium and Helium-3. Physical Review Letters, 114, Article ID: 013003.

[15] Chekhovich, E.A., Hopkinson, M., Skolnick, M.S. and Tartakovskii, A.I. (2015) Suppression of Nuclear Spin Bath Fluctuations in Self-Assembled Quantum Dots Induced by Inhomogeneous Strain. Nature Communications, 6, Article No. 6348. https://doi.org/10.1038/ncomms7348

[16] Dong, Y., Dong, L., Gong, M. and Pu, H. (2015) Dynamical Phases in Quenched Spin-Orbit-Coupled Degenerate Fermi Gas. Nature Communications, 6, Article No. 6103. https://doi.org/10.1038/ncomms7103

[17] Koizumi, H., Okazaki, A., Ghantous, M.A. and Tachiki, M. (2014) Supercurrent Flow through the Network of Spin-Vortices in Cuprates. Journal of Superconductivity and Novel Magnetism, 27, 2435-2446. https://doi.org/10.1007/s10948-014-2626-9

[18] Tan, Z.B., Cox, D., Nieminen, T., Lähteenmäki, P., Golubev, D., Lesovik, G.B. and Hakonen, P.J. (2015) Cooper Pair Splitting by Means of Graphene Quantum Dots. Physical Review Letters, 114, Article ID: 096602.

[19] Tan, D., Weber, S.J., Siddiqi, I., Mølmer, K. and Murch, K.W. (2015) Prediction and Retrodiction for a Continuously Monitored Super-Conducting Qubit. Physical Review Letters, 114, Article ID: 090403.

[20] Murmann, S., Bergschneider, A., Klinkhamer, V.M., Zürn, G., Lompe, T. and Jochim, S. (2015) Two Fermions in a Double Well: Exploring a Fundamental Building Block of the Hubbard Model. Physical Review Letters, 114, Article ID: 080402.

[21] Giraud, O., Braun, D., Baguette, D., Bastin, T. and Martin, J. (2015) Tensor Representation of Spin States. Physical Review Letters, 114, Article ID: 080401.

[22] Valderrama, M.P. and Phillips, D.R. (2015) Power Counting of Contact-Range Currents in Effective Field Theory. Physical Review Letters, 114, Article ID: 082502.

[23] Phillips, D.R., Samart, D. and Schat, C. (2015) Parity-Violating Nucleon-Nucleon Force in the 1/Nc Expansion. Physical Review Letters, 114, Article ID: 062301.

[24] Stetcu, I., Bertulani, C.A., Bulgac, A., Magierski, P. and Roche, K.J. (2015) Relativistic Coulomb Excitation within the Time Dependent Superfluid Local Density Approximation. Physical Review Letters, 114, Article ID: 012701.

[25] Borsanyi, S., Durr, S., Fodor, Z., Hoelbling, C., Katz, S.D., Krieg, S., et al. (2015) Ab Initio Calculation of the Neutron-Proton Mass Difference. Science, 347, 1452-1455. https://doi.org/10.1126/science.1257050

[26] Freedman, R. and Young, H. (2004) Sears and Zemanskey's University Physics with Modern Physics. 11th Edition, Benjamin-Cummings Pub. Co., San Francisco, 1633-1634.

[27] Abell, G., Corwin, H. and Olowin, R. (1989) A Catalogue of Rich Clusters of Galax- 
ies. Astrophysical Journal Supplement, 70, 1-138.

[28] Struble, M. and Rood, H. (1999) A Compilation of Redshifts and Velocity Dispersions for ACO Clusters. The Astrophysical Journal, 125, 35-71.

https://doi.org/10.1086/313274 